KYUNGPOOK Math. J. 55(2015), 313-326

http://dx.doi.org/10.5666/KMJ.2015.55.2.313

pISSN 1225-6951 eISSN 0454-8124

(C) Kyungpook Mathematical Journal

\title{
A Fixed Point Approach to Stability of Quintic Functional Equations in Modular Spaces
}

\author{
Mohammad Bagher Ghaemi \\ Department of Mathematics, Iran University of Science and Technology, Narmak, \\ Tehran, Iran \\ e-mail : mghaemi@iust.ac.ir \\ Mehdi ChOubin \\ Department of Mathematics, Velayat University, Iranshahr, Iran \\ e-mail : m.choubin@gmail.com \\ GHADIR SADEGHI \\ Department of Mathematics and Computer Sciences, Hakim Sabzevari University, \\ Sabzevar, P. O. Box 397, Iran \\ e-mail : ghadir54@gmail.com \\ MadjID Eshaghi GordJi* \\ Department of Mathematics, Semnan University, Semnan, Iran \\ e-mail : meshaghi@semnan.ac.ir; madjid.eshaghi@gmail.com
}

ABSTRACT. In this paper, we present a fixed point method to prove generalized HyersUlam stability of the systems of quadratic-cubic functional equations with constant coefficients in modular spaces.

\section{Introduction}

The stability problem of functional equations started with the following question concerning stability of group homomorphisms proposed by S.M. Ulam [58] during a talk before a Mathematical Colloquium at the University of Wisconsin, Madison, in 1940.

Let $\left(G_{1},.\right)$ be a group and $\left(G_{2}, *\right)$ be a metric group with the metric $d(\cdot, \cdot)$.

* Corresponding Author.

Received August 3, 2012; accepted August 2, 2013.

2010 Mathematics Subject Classification: Primary 39B52; Secondary 39B72, 47H09.

Key words and phrases: stability, quintic functional equation, fixed point, modular space. 
Given $\epsilon>0$, does there exist a $\delta>0$ such that, if a mapping $h: G_{1} \longrightarrow G_{2}$ satisfies the inequality $d(h(x . y), h(x) * h(y))<\delta$ for all $x, y \in G_{1}$, then there exists a homomorphism $H: G_{1} \longrightarrow G_{2}$ with $d(h(x), H(x))<\epsilon$ for all $x \in G_{1}$ ?

In 1941, Hyers [24] gave a first affirmative answer to the question of Ulam for Banach spaces as follows:

If $E$ and $E^{\prime}$ are Banach spaces and $f: E \longrightarrow E^{\prime}$ is a mapping for which there is $\varepsilon>0$ such that $\|f(x+y)-f(x)-f(y)\| \leq \varepsilon$ for all $x, y \in E$, then there is a unique additive mapping $L: E \longrightarrow E^{\prime}$ such that $\|f(x)-L(x)\| \leq \varepsilon$ for all $x \in E$.

Hyers' Theorem was generalized by Aoki [2] for additive mappings and by Rassias [51] for linear mappings by considering an unbounded Cauchy difference, respectively.

The paper of Rassias [51] has provided a lot of influence in the development of what we now call the generalized Hyers-Ulam stability or as Hyers-Ulam-Rassias stability of functional equations. In 1994, a generalization of the Rassias theorem was obtained by Găvruta [10] by replacing the unbounded Cauchy difference by a general control function in the spirit of Rassias' approach. For more details about the results concerning such problems, the reader refer to $[3,5,6,9,11,19,25,26$, 27, 28, 31, 32, 33, 36, 52] and [46]-[53]. Recently, Sadeghi [54] presented a fixed point method to prove generalized Hyers-Ulam stability of the generalized Jensen functional equation $f(r x+s y)=r g(x)+s h(x)$ in modular spaces.

The functional equation

$$
f(x+y)+f(x-y)=2 f(x)+2 f(y)
$$

is related to a symmetric bi-additive function $[1,34]$. It is natural that this equation is called a quadratic functional equation. In particular, every solution of the quadratic equation (1.1) is called a quadratic function. The Hyers-Ulam stability problem for the quadratic functional equation was solved by Skof [56]. In [5], Czerwik proved the Hyers-Ulam-Rassias stability of the equation (1.1). Eshaghi Gordji and Khodaei [20] obtained the general solution and the generalized Hyers-UlamRassias stability of the following quadratic functional equation: for all $a, b \in \mathbb{Z} \backslash\{0\}$ with $a \neq \pm 1, \pm b$,

$$
f(a x+b y)+f(a x-b y)=2 a^{2} f(x)+2 b^{2} f(y) .
$$

Jun and Kim [29] introduced the following cubic functional equation:

$$
f(2 x+y)+f(2 x-y)=2 f(x+y)+2 f(x-y)+12 f(x)
$$

and they established the general solution and the generalized Hyers-Ulam-Rassias stability for the functional equation (1.3). Jun et al. [30] investigated the solution and the Hyers-Ulam stability for the cubic functional equation

$$
f(a x+b y)+f(a x-b y)=a b^{2}(f(x+y)+f(x-y))+2 a\left(a^{2}-b^{2}\right) f(x),
$$


where $a, b \in \mathbb{Z} \backslash\{0\}$ with $a \neq \pm 1, \pm b$. For other cubic functional equations, see [43].

Lee et. al. [39] considered the following functional equation:

$$
f(2 x+y)+f(2 x-y)=4 f(x+y)+4 f(x-y)+24 f(x)-6 f(y)
$$

In fact, they proved that a function $f$ between two real vector spaces $X$ and $Y$ is a solution of the equation (1.5) if and only if there exists a unique symmetric bi-quadratic function $B_{2}: X \times X \longrightarrow Y$ such that $f(x)=B_{2}(x, x)$ for all $x \in X$. The bi-quadratic function $B_{2}$ is given by

$$
B_{2}(x, y)=\frac{1}{12}(f(x+y)+f(x-y)-2 f(x)-2 f(y)) .
$$

Obviously, the function $f(x)=c x^{4}$ satisfies the functional equation (1.5), which is called the quartic functional equation. For other quartic functional equations, see [4].

Ebadian et al. [7] considered the generalized Hyers-Ulam stability of the following systems of the additive-quartic functional equations:

$$
\left\{\begin{array}{l}
f\left(x_{1}+x_{2}, y\right)=f\left(x_{1}, y\right)+f\left(x_{2}, y\right) \\
f\left(x, 2 y_{1}+y_{2}\right)+f\left(x, 2 y_{1}-y_{2}\right) \\
\quad=4 f\left(x, y_{1}+y_{2}\right)+4 f\left(x, y_{1}-y_{2}\right)+24 f\left(x, y_{1}\right)-6 f\left(x, y_{2}\right)
\end{array}\right.
$$

and the quadratic-cubic functional equations:

$$
\left\{\begin{array}{c}
f\left(x, 2 y_{1}+y_{2}\right)+f\left(x, 2 y_{1}-y_{2}\right) \\
\quad=2 f\left(x, y_{1}+y_{2}\right)+2 f\left(x, y_{1}-y_{2}\right)+12 f\left(x, y_{1}\right) \\
f\left(x, y_{1}+y_{2}\right)+f\left(x, y_{1}-y_{2}\right)=2 f\left(x, y_{1}\right)+2 f\left(x, y_{2}\right) .
\end{array}\right.
$$

For more details about the results concerning mixed type functional equations, the readers refer to $[13,16,17]$ and $[18]$.

Recently, Ghaemi et. al. [12] investigated the the stability of the following systems of quadratic-cubic functional equations:

$$
\left\{\begin{array}{l}
f\left(a x_{1}+b x_{2}, y\right)+f\left(a x_{1}-b x_{2}, y\right)=2 a^{2} f\left(x_{1}, y\right)+2 b^{2} f\left(x_{2}, y\right) \\
f\left(x, a y_{1}+b y_{2}\right)+f\left(x, a y_{1}-b y_{2}\right) \\
\quad=a b^{2}\left(f\left(x, y_{1}+y_{2}\right)+f\left(x, y_{1}-y_{2}\right)\right)+2 a\left(a^{2}-b^{2}\right) f\left(x, y_{1}\right)
\end{array}\right.
$$

in PN-spaces, where $a, b \in \mathbb{Z} \backslash\{0\}$ with $a \neq \pm 1, \pm b$. The function $f: R \times R \rightarrow R$ given by $f(x, y)=c x^{2} y^{3}$ is a solution of the system (1.8). In particular, letting $y=x$, we get a quintic function $g: R \rightarrow R$ in one variable given by $g(x):=$ $f(x, x)=c x^{5}$.

The proof of the following propositions is evident.

Proposition 1.1. Let $X$ and $Y$ be real linear spaces. If a function $f: X \times X \longrightarrow Y$ satisfies the system (1.8), then $f(\lambda x, \mu y)=\lambda^{2} \mu^{3} f(x, y)$ for all $x, y \in X$ and rational 
numbers $\lambda, \mu$.

In this paper, by using some ideas of $[14,54]$, we investigate the generalized Hyers-Ulame stability of a quintic mappings from linear spaces into modular spaces. The theory of modulars on linear spaces and the corresponding theory of modular linear spaces were founded by Nakano [44] and were intensively developed by Amemiya, Koshi, Shimogaki, Yamamuro [37, 59] and others. Further and the most complete development of these theories are due to Orlicz, Mazur, Musielak, Luxemburg, Turpin $[40,42,57]$ and their collaborators. In the present time the theory of modulars and modular spaces is extensively applied, in particular, in the study of various Orlicz spaces [45] and interpolation theory [38, 41], which in their turn have broad applications [42]. The importance for applications consists in the richness of the structure of modular function spaces, that-besides being Banach spaces (or $F$-spaces in more general setting) - are equipped with modular equivalent of norm or metric notions.

Definition 1.2. Let $X$ be an arbitrary vector space.

(a) A functional $\rho: X \rightarrow[0, \infty]$ is called a modular if for arbitrary $x, y \in X$,

(i) $\rho(x)=0$ if and only if $x=0$,

(ii) $\rho(\alpha x)=\rho(x)$ for every scaler $\alpha$ with $|\alpha|=1$,

(iii) $\rho(\alpha x+\beta y) \leq \rho(x)+\rho(y)$ if and only if $\alpha+\beta=1$ and $\alpha, \beta \geq 0$,

(b) if (iii) is replaced by

(iii) $)^{\prime} \rho(\alpha x+\beta y) \leq \alpha \rho(x)+\beta \rho(y)$ if and only if $\alpha+\beta=1$ and $\alpha, \beta \geq 0$,

then we say that $\rho$ is a convex modular. A modular $\rho$ defines a corresponding modular space, i.e., the vector space $X_{\rho}$ given by

$$
X_{\rho}=\{x \in X: \quad \rho(\lambda x) \rightarrow 0 \text { as } \lambda \rightarrow 0\} .
$$

Let $\rho$ be a convex modular, the modular space $X_{\rho}$ can be equipped with a norm called the Luxemburg norm, defined by

$$
\|x\|_{\rho}=\inf \left\{\lambda>0 \quad ; \quad \rho\left(\frac{x}{\lambda}\right) \leq 1\right\} .
$$

A function modular is said to satisfy the $\Delta_{2}$-condition if there exists $\kappa>0$ such that $\rho(2 x) \leq \kappa \rho(x)$ for all $x \in X_{\rho}$.

Definition 1.3. Let $\left\{x_{n}\right\}$ and $x$ be in $X_{\rho}$. Then

(i) the sequence $\left\{x_{n}\right\}$, with $x_{n} \in X_{\rho}$, is $\rho$-convergent to $x$ and write $x_{n} \stackrel{\rho}{\longrightarrow} x$ if $\rho\left(x_{n}-x\right) \rightarrow 0$ as $n \rightarrow \infty$.

(ii) The sequence $\left\{x_{n}\right\}$, with $x_{n} \in X_{\rho}$, is called $\rho$-Cauchy if $\rho\left(x_{n}-x_{m}\right) \rightarrow 0$ as $n, m \rightarrow \infty$.

(iii) A subset $\mathcal{S}$ of $X_{\rho}$ is called $\rho$-complete complete if and only if any $\rho$-Cauchy sequence is $\rho$-convergent to an element of $\mathcal{S}$. 
The modular $\rho$ has the Fatou property if and only if $\rho(x) \leq \liminf _{n \rightarrow \infty} \rho\left(x_{n}\right)$ whenever the sequence $\left\{x_{n}\right\}$ is $\rho$-convergent to $x$.

Remark 1.4. Note that $\rho$ is an increasing function. Suppose $0<a<b$, then property (iii) of Definition 1.2 with $y=0$ shows that $\rho(a x)=\rho\left(\frac{a}{b} b x\right) \leq \rho(b x)$ for all $x \in X$. Moreover, if $\rho$ is a convex modular on $X$ and $|\alpha| \leq 1$, then $\rho(\alpha x) \leq \alpha \rho(x)$ and also $\rho(x) \leq \frac{1}{2} \rho(2 x)$ for all $x \in X$.

A convex function $\varphi$ defined on the interval $[0, \infty)$, nondecreasing and continuous for $\alpha \geq 0$ and such that $\varphi(0)=0, \varphi(\alpha)>0$ for $\alpha>0, \varphi(\alpha) \rightarrow \infty$ as $\alpha \rightarrow \infty$, is called an Orlicz function. The Orlicz function $\varphi$ satisfies the $\Delta_{2}$-condition if there exists $\kappa>0$ such that $\varphi(2 \alpha) \leq \varphi(\alpha)$ for all $\alpha>0$. Let $(\Omega, \Sigma, \mu)$ be a measure space. Let us consider the space $L^{0}(\mu)$ consisting of all measurable real-valued (or complex-valued) functions on $\Omega$. Define for every $f \in L^{0}(\mu)$ the Orlicz modular $\rho_{\varphi}(f)$ by the formula

$$
\rho_{\varphi}(f)=\int_{\Omega} \varphi(|f|) d \mu .
$$

The associated modular function space with respect to this modular is called an Orlicz space, and will be denoted by $L^{\varphi}(\Omega, \mu)$ or briefly $L^{\varphi}$. In other words,

$$
L^{\varphi}=\left\{f \in L^{0}(\mu) \quad \mid \quad \rho_{\varphi}(\lambda f) \rightarrow 0 \text { as } \lambda \rightarrow 0\right\}
$$

or equivalently as

$$
L^{\varphi}=\left\{f \in L^{0}(\mu) \quad \mid \quad \rho_{\varphi}(\lambda f)<\infty \text { for some } \lambda>0\right\} .
$$

It is known that the Orlicz space $L^{\varphi}$ is $\rho_{\varphi}$-complete. Moreover, $\left(L^{\varphi},\|\cdot\|_{\rho_{\varphi}}\right)$ is a Banach space, where the Luxemburg norm $\|\cdot\|_{\rho_{\varphi}}$ is defined as follows

$$
\|f\|_{\rho_{\varphi}}=\inf \left\{\lambda>0: \int_{\Omega} \varphi\left(\frac{|f|}{\lambda}\right) d \mu \leq 1\right\} .
$$

Moreover, if $\mathfrak{L}$ is the space of sequences $x=\left\{x_{i}\right\}_{i=1}^{\infty}$ with real or complex terms $x_{i}$, $\varphi=\left\{\varphi_{i}\right\}_{i=1}^{\infty}, \varphi_{i}$ are Orlicz functions and $\varrho_{\varphi}(x)=\sum_{i=1}^{\infty} \varphi_{i}\left(\left|x_{i}\right|\right)$, we shall write $\ell^{\varphi}$ in place of $L^{\varphi}$. The space $\ell^{\varphi}$ is called the generalized Orlicz sequence space. The motivation for the study of modular spaces (and Orlicz spaces) and many examples are detailed in $[41,42,44,45]$.

\section{Main Results}

Throughout this paper, we assume that $\rho$ is a convex modular on $X$ with the Fatou property such that satisfies the $\Delta_{2}$-condition with $0<\kappa \leq 2$. In this section, we establish the conditional stability of quintic functional equations.

Theorem 2.1. Let $E$ be a real or complex linear space and let $X_{\rho}$ be a $\rho$-complete 
modular space. Suppose $f: E \times E \rightarrow X_{\rho}$ satisfies the condition $f(0, y)=0$ and an inequality of the form

$$
\begin{aligned}
\rho\left(f\left(a x_{1}+b x_{2}, y\right)+f\left(a x_{1}-b x_{2}, y\right)-2 a^{2} f\left(x_{1}, y\right)\right. & \\
\left.-2 b^{2} f\left(x_{2}, y\right)\right) & \leq \phi\left(x_{1}, x_{2}, y\right), \\
\rho\left(f\left(x, a y_{1}+b y_{2}\right)+f\left(x, a y_{1}-b y_{2}\right)-a b^{2} f\left(x, y_{1}+y_{2}\right)\right. & \\
\left.-a b^{2} f\left(x, y_{1}-y_{2}\right)-2 a\left(a^{2}-b^{2}\right) f\left(x, y_{1}\right)\right) & \leq \psi\left(x, y_{1}, y_{2}\right),
\end{aligned}
$$

where $\phi, \psi: E \times E \times E \rightarrow[0, \infty)$ is a given function such that

$$
\phi(a x, 0, a y) \leq a^{5} L \phi(x, 0, y), \quad \psi\left(a^{2} x, a y, 0\right) \leq a^{5} L \psi(a x, y, 0),
$$

and has the property

$$
\lim _{n \rightarrow \infty} \frac{\phi\left(a^{n} x_{1}, a^{n} x_{2}, a^{n} y\right)}{a^{5 n}}=\frac{\psi\left(a^{n} x, a^{n} y_{1}, a^{n} y_{2}\right)}{a^{5 n}}=0,
$$

for all $x, x_{1}, x_{2}, y, y_{1}, y_{2} \in E$ and a constant $0<L<1$. Then there exists a unique quintic function $j: E \times E \rightarrow X_{\rho}$ satisfying the system (1.8) and

$$
\rho(j(x, y)-f(x, y)) \leq \frac{1}{1-L}\left(\frac{1}{a^{2}} \phi(x, 0, y)+\frac{1}{a^{5}} \psi(a x, y, 0)\right),
$$

for all $x, y \in E$.

Proof. Putting $x_{1}=2 x$ and $x_{2}=0$ and replacing $y$ by $2 y$ in (2.1), we get

$$
\rho\left(2 f(2 a x, 2 y)-2 a^{2} f(2 x, 2 y)\right) \leq \phi(2 x, 0,2 y)
$$

for all $x, y \in E$. Putting $y_{1}=2 y$ and $y_{2}=0$ and replacing $x$ by $2 a x$ in (2.2), we get

$$
\rho\left(2 f(2 a x, 2 a y)-2 a^{3} f(2 a x, 2 y)\right) \leq \psi(2 a x, 2 y, 0)
$$

for all $x, y \in E$. Thus by (2.3) and (2.4) we have

$$
\begin{aligned}
& \rho\left(a^{-3} f(2 a x, 2 a y)-a^{2} f(2 x, 2 y)\right) \\
& \leq \frac{1}{2} \rho\left(2 a^{-3} f(2 a x, 2 a y)-2 f(2 a x, 2 y)\right)+\frac{1}{2} \rho\left(2 f(2 a x, 2 y)-2 a^{2} f(2 x, 2 y)\right) \\
& \leq \frac{1}{2 a^{3}} \rho\left(2 f(2 a x, 2 a y)-2 a^{3} f(2 a x, 2 y)\right)+\frac{1}{2} \rho\left(2 f(2 a x, 2 y)-2 a^{2} f(2 x, 2 y)\right) \\
& \leq \frac{1}{2 a^{3}} \psi(2 a x, 2 y, 0)+\frac{1}{2} \phi(2 x, 0,2 y),
\end{aligned}
$$

for all $x, y \in E$. By last inequality we get

$$
\rho\left(a^{-5} f(2 a x, 2 a y)-f(2 x, 2 y)\right) \leq \frac{1}{2 a^{5}} \psi(2 a x, 2 y, 0)+\frac{1}{2 a^{2}} \phi(2 x, 0,2 y) .
$$


Replacing $x, y$ by $\frac{x}{2}, \frac{y}{2}$ in $(2.5)$, we have

$$
\rho\left(a^{-5} f(a x, a y)-f(x, y)\right) \leq \frac{1}{2 a^{5}} \psi(a x, y, 0)+\frac{1}{2 a^{2}} \phi(x, 0, y),
$$

for all $x, y \in E$.

We now consider the set

$$
\mathcal{M}=\left\{h: E \times E \rightarrow X_{\rho}, \quad h(0, y)=0 \text { for all } y \in E\right\}
$$

and introduce the convex modular $\widetilde{\rho}$ on $\mathcal{M}$ as follows,

$$
\widetilde{\rho}(h)=\inf \{c>0: \rho(h(x, y)) \leq c \Phi(x, y)\},
$$

where $\Phi(x, y):=\frac{1}{a^{5}} \psi(a x, y, 0)+\frac{1}{a^{2}} \phi(x, 0, y)$. It is sufficient to show that $\widetilde{\rho}$ satisfies the following condition

$$
\widetilde{\rho}(\alpha g+\beta h) \leq \alpha \widetilde{\rho}(g)+\beta \widetilde{\rho}(h)
$$

if $\alpha+\beta=1$ and $\alpha, \beta \geq 0$. Let $\varepsilon>0$ be given. Then there exist $c_{1}>0$ and $c_{2}>0$ such that

$$
c_{1} \leq \widetilde{\rho}(g)+\varepsilon ; \quad \rho(g(x, y)) \leq c_{1} \Phi(x, y)
$$

and

$$
c_{2} \leq \widetilde{\rho}(h)+\varepsilon ; \quad \rho(h(x, y)) \leq c_{2} \Phi(x, y) .
$$

If $\alpha+\beta=1$ and $\alpha, \beta \geq 0$, then we get

$$
\rho(\alpha g(x, y)+\beta h(x, y)) \leq \alpha \rho(g(x, y))+\beta \rho(h(x, y)) \leq\left(\alpha c_{1}+\beta c_{2}\right) \Phi(x, y),
$$

whence

$$
\widetilde{\rho}(\alpha g+\beta h) \leq \alpha \widetilde{\rho}(g)+\beta \widetilde{\rho}(h)+(\alpha+\beta) \varepsilon
$$

Hence, we have

$$
\widetilde{\rho}(\alpha g+\beta h) \leq \alpha \widetilde{\rho}(g)+\beta \widetilde{\rho}(h) .
$$

Moreover, $\widetilde{\rho}$ satisfies the $\Delta_{2}$-condition with $0<\kappa \leq 2$. Indeed for $\varepsilon>0$ given, there exists $c>0$ such that

$$
c \leq \widetilde{\rho}(h)+\varepsilon ; \quad \rho(h(x, y)) \leq c \Phi(x, y) .
$$

Since $\rho$ satisfies the $\Delta_{2}$-condition, we have

$$
\rho(2 h(x, y)) \leq \kappa \rho(h(x, y)) \leq \kappa c \Phi(x, y)
$$

therefore $\widetilde{\rho}(2 h) \leq \kappa c \leq \kappa \widetilde{\rho}(h)+\kappa \varepsilon$. Thus $\widetilde{\rho}$ satisfies the $\Delta_{2}$-condition.

Let $\left\{h_{n}\right\}$ be a $\widetilde{\rho}$-Cauchy sequence in $\mathcal{M}_{\widetilde{\rho}}$ and let $\varepsilon>0$ be given. There exists a positive integer $n_{0} \in \mathbb{N}$ such that $\widetilde{\rho}\left(h_{n}-h_{m}\right) \leq \varepsilon$ for all $n, m \geq n_{0}$. Now by considering the definition of the modular $\widetilde{\rho}$, we see that

$$
\rho\left(h_{n}(x, y)-h_{m}(x, y)\right) \leq \varepsilon \Phi(x, y)
$$


for all $x, y \in E$ and $n, m \geq n_{0}$. If $x$ and $y$ are arbitrary given points of $E$, (2.7) implies that $\left\{h_{n}(x, y)\right\}$ is a $\rho$-Cauchy sequence in $X_{\rho}$. Since $X_{\rho}$ is $\rho$-complete, so $\left\{h_{n}(x, y)\right\}$ is $\rho$-convergent in $X_{\rho}$, for all $x, y \in E$. Hence, we can define a function $h: E \times E \rightarrow X_{\rho}$ by

$$
h(x, y)=\lim _{n \rightarrow \infty} h_{n}(x, y),
$$

for any $x$ and $y \in E$. Let $m$ increase to infinity, then (2.7) implies that

$$
\widetilde{\rho}\left(h_{n}-h\right) \leq \varepsilon
$$

for all $n \geq n_{0}$, since $\rho$ has the Fatou property. Thus $\left\{h_{n}\right\}$ is $\widetilde{\rho}$-convergent sequence in $\mathcal{M}_{\tilde{\rho}}$. Therefore $\mathcal{M}_{\tilde{\rho}}$ is $\widetilde{\rho}$-complete.

Now, we consider the function $\mathcal{T}: \mathcal{M}_{\tilde{\rho}} \rightarrow \mathcal{M}_{\tilde{\rho}}$ defined by

$$
\mathcal{T} h(x, y):=a^{-5} h(a x, a y)
$$

for all $h \in \mathcal{M}_{\tilde{\rho}}$. Let $g, h \in \mathcal{M}_{\tilde{\rho}}$ and let $c \in[0, \infty]$ be an arbitrary constant with $\widetilde{\rho}(g-h) \leq c$. From the definition of $\widetilde{\rho}$, we have

$$
\rho(g(x, y)-h(x, y)) \leq c \Phi(x, y)
$$

for all $x, y \in E$. By the assumption and the last inequality, we get

$$
\begin{aligned}
\rho(\mathcal{T} g(x, y)-\mathcal{T} h(x, y)) & =\rho\left(a^{-5} g(a x, a y)-a^{-5} h(a x, a y)\right) \\
& \leq \frac{1}{a^{5}} \rho(g(a x, a y)-h(a x, a y)) \\
& \leq \frac{1}{a^{5}} c \Phi(a x, a y) \\
& \leq L c \Phi(x, y),
\end{aligned}
$$

for all $x \in E$. Hence, $\widetilde{\rho}(\mathcal{T} g-\mathcal{T} h) \leq L \widetilde{\rho}(g-h)$, for all $g, h \in \mathcal{M}_{\tilde{\rho}}$ that is, $\mathcal{T}$ is a $\tilde{\rho}$-strict contraction. We show that the $\widetilde{\rho}$-strict mapping $\mathcal{T}$ satisfies the conditions of Theorem 3.4 of [35].

By replacing $x, y$ by $a x$, ay in (2.6), we get

$$
\rho\left(a^{-5} f\left(a^{2} x, a^{2} y\right)-f(a x, a y)\right) \leq \frac{1}{2 a^{5}} \psi\left(a^{2} x, a y, 0\right)+\frac{1}{2 a^{2}} \phi(a x, 0, a y),
$$

and so

$$
\rho\left(a^{-2(5)} f\left(a^{2} x, a^{2} y\right)-a^{-5} f(a x, a y)\right) \leq \frac{1}{2 a^{2(5)}} \psi\left(a^{2} x, a y, 0\right)+\frac{1}{2 a^{2+5}} \phi(a x, 0, a y),
$$

for all $x, y \in E$. Since $\rho$ is convex modular which satisfies the $\Delta_{2}$-condition, and 
$\kappa / 2 \leq 1$, by $(2.6)$ and last inequality we obtain

$$
\begin{aligned}
& \rho\left(a^{-2(5)} f\left(a^{2} x, a^{2} y\right)-f(x, y)\right) \\
& \leq \frac{1}{2} \rho\left(2 a^{-2(5)} f\left(a^{2} x, a^{2} y\right)-2 a^{-5} f(a x, a y)\right)+\frac{1}{2} \rho\left(2 a^{-5} f(a x, a y)-2 f(x, y)\right) \\
& \leq \frac{\kappa}{2} \rho\left(a^{-2(5)} f\left(a^{2} x, a^{2} y\right)-a^{-5} f(a x, a y)\right)+\frac{\kappa}{2} \rho\left(a^{-5} f(a x, a y)-f(x, y)\right) \\
& \leq\left\{\frac{1}{2 a^{2(5)}} \psi\left(a^{2} x, a y, 0\right)+\frac{1}{2 a^{5}} \psi(a x, y, 0)\right\}+\left\{\frac{1}{2 a^{2+5}} \phi(a x, 0, a y)+\frac{1}{2 a^{2}} \phi(x, 0, y)\right\},
\end{aligned}
$$

for all $x, y \in E$. By mathematical induction, we can easily see that

$$
\begin{aligned}
& \rho\left(\frac{f\left(a^{n} x, a^{n} y\right)}{a^{5 n}}-f(x, y)\right) \\
& \leq \sum_{i=1}^{n} \frac{1}{2 a^{5 i}} \psi\left(a^{i} x, a^{i-1} y, 0\right)+\sum_{i=1}^{n} \frac{1}{2 a^{2+5(i-1)}} \phi\left(a^{i-1} x, 0, a^{i-1} y\right) \\
& \leq \psi(a x, y, 0) \sum_{i=1}^{n} \frac{a^{5(i-1)}}{2 a^{5 i}} L^{i-1}+\phi(x, 0, y) \sum_{i=1}^{n} \frac{a^{5(i-1)}}{2 a^{2+5(i-1)}} L^{i-1} \\
& \leq \frac{1}{2(1-L)} \Phi(x, y)
\end{aligned}
$$

for all $x, y \in E$. Next, we assert that $\left.\delta_{\widetilde{\rho}}(f)=\sup \left\{\widetilde{\rho}\left(\mathcal{T}^{n}(f)-\mathcal{T}^{m}(f)\right) ; n, m \in \mathbb{N}\right)\right\}<$ $\infty$. It follows from inequality (2.9) that

$$
\begin{aligned}
& \rho\left(\frac{f\left(a^{n} x, a^{n} y\right)}{a^{n}}-\frac{f\left(a^{m} x, a^{m} y\right)}{a^{m}}\right) \\
& \leq \frac{1}{2} \rho\left(2 \frac{f\left(a^{n} x, a^{n} y\right)}{a^{n}}-2 f(x, y)\right)+\frac{1}{2} \rho\left(2 \frac{f\left(a^{m} x, a^{m} y\right)}{a^{m}}-2 f(x, y)\right) \\
& \leq \frac{\kappa}{2} \rho\left(\frac{f\left(a^{n} x, a^{n} y\right)}{a^{n}}-f(x, y)\right)+\frac{\kappa}{2} \rho\left(\frac{f\left(a^{m} x, a^{m} y\right)}{a^{m}}-f(x, y)\right) \\
& \leq \frac{1}{(1-L)} \Phi(x, y),
\end{aligned}
$$

for every $x, y \in E$ and $n, m \in \mathbb{N}$, which implies that

$$
\widetilde{\rho}\left(\mathcal{T}^{n}(f)-\mathcal{T}^{m}(f)\right) \leq \frac{1}{1-L},
$$

for all $n, m \in \mathbb{N}$. By the definition of $\delta_{\widetilde{\rho}}(f)$, we have $\delta_{\widetilde{\rho}}(f)<\infty$. Lemma 3.3 of [35] shows that $\left\{\mathcal{T}^{n}(f)\right\}$ is $\widetilde{\rho}$-converges to $j \in \mathcal{M}_{\widetilde{\rho}}$. Since $\rho$ has the Fatou property inequality (2.9), gives $\widetilde{\rho}(\mathcal{T} j-f)<\infty$.

If we replace $m$ by $n+1$ in inequality (2.10), then we obtain

$$
\rho\left(\frac{f\left(a^{n+1} x, a^{n+1} y\right)}{a^{n+1}}-\frac{f\left(a^{n} x, a^{n} y\right)}{a^{n}}\right) \leq \frac{1}{(1-L)} \Phi(x, y)
$$


for all $x, y \in E$. Therefore $\widetilde{\rho}(\mathcal{T}(j)-j) \leq(1 / 1-L)<\infty$. It follows from [35, Theorem 3.4] that $\widetilde{\rho}$-limit of $\left\{\mathcal{T}^{n}(f)\right\}$ i.e., $j \in \mathcal{M}_{\tilde{\rho}}$ is fixed point of map $\mathcal{T}$. If we replace $x_{1}, x_{2}$ and $y$ by $a^{n} x_{1}, a^{n} x_{2}$ and $a^{n} y$ in inequality (2.1), respectively, then we obtain

$$
\begin{aligned}
& \rho\left(\frac{f\left(a^{n}\left(a x_{1}+b x_{2}\right), a^{n} y\right)}{a^{5 n}}+\frac{f\left(a^{n}\left(a x_{1}-b x_{2}\right), a^{n} y\right)}{a^{5 n}}\right. \\
& \left.\quad-2 a^{2} \frac{f\left(a^{n} x_{1}, a^{n} y\right)}{a^{5 n}}-2 b^{2} \frac{f\left(a^{n} x_{2}, a^{n} y\right)}{a^{5 n}}\right) \\
& \leq \frac{1}{a^{5 n}} \rho\left(f\left(a^{n}\left(a x_{1}+b x_{2}\right), a^{n} y\right)+f\left(a^{n}\left(a x_{1}-b x_{2}\right), a^{n} y\right)\right. \\
& \left.\quad-2 a^{2} f\left(a^{n} x_{1}, a^{n} y\right)-2 b^{2} f\left(a^{n} x_{2}, a^{n} y\right)\right) \\
& \leq \frac{1}{a^{5 n}} \phi\left(a^{n} x_{1}, a^{n} x_{2}, a^{n} y\right),
\end{aligned}
$$

and similarly by replacing $x, y_{1}$ and $y_{2}$ by $a^{n} x, a^{n} y_{1}$ and $a^{n} y_{2}$ in inequality (2.2), respectively, we get

$$
\begin{aligned}
& \rho\left(\frac{f\left(a^{n} x, a^{n}\left(a y_{1}+b y_{2}\right)\right)}{a^{5 n}}+\frac{f\left(a^{n} x, a^{n}\left(a y_{1}-b y_{2}\right)\right)}{a^{5 n}}-a b^{2} \frac{f\left(a^{n} x, a^{n}\left(y_{1}+y_{2}\right)\right)}{a^{5 n}}\right. \\
& \left.-a b^{2} \frac{f\left(a^{n} x, a^{n}\left(y_{1}-y_{2}\right)\right)}{a^{5 n}}-2 a\left(a^{2}-b^{2}\right) \frac{f\left(a^{n} x, a^{n} y_{1}\right)}{a^{5 n}}\right) \leq \frac{1}{a^{5 n}} \psi\left(a^{n} x, a^{n} y_{1}, a^{n} y_{2}\right),
\end{aligned}
$$

for all $x, x_{1}, x_{2}, y, y_{1}, y_{2} \in E$. Taking the limit, we deduce that $j$ satisfying the system (1.8), that is, $j$ is quintic. It follows from inequality (2.9) that

$$
\widetilde{\rho}(j-f) \leq \frac{1}{2(1-L)} .
$$

If $j^{*}$ is another fixed point of $\mathcal{T}$, then

$$
\begin{aligned}
\widetilde{\rho}\left(j-j^{*}\right) & \leq \frac{1}{2} \widetilde{\rho}(2 \mathcal{T}(j)-2 f)+\frac{1}{2} \widetilde{\rho}\left(2 \mathcal{T}\left(j^{*}\right)-2 f\right) \\
& \leq \frac{\kappa}{2} \widetilde{\rho}(\mathcal{T}(j)-f)+\frac{\kappa}{2} \widetilde{\rho}\left(\mathcal{T}\left(j^{*}\right)-f\right) \leq \frac{\kappa}{2(1-L)}<\infty .
\end{aligned}
$$

Since $\mathcal{T}$ is $\widetilde{\rho}$-strict contraction, we get

$$
\widetilde{\rho}\left(j-j^{*}\right)=\widetilde{\rho}\left(\mathcal{T}(j)-\mathcal{T}\left(j^{*}\right)\right) \leq L \widetilde{\rho}\left(j-j^{*}\right),
$$

which implies that $\widetilde{\rho}\left(j-j^{*}\right)=0$ or $j=j^{*}$, since $\widetilde{\rho}\left(j-j^{*}\right)<\infty$. This prove the uniqueness of $j$.

Corollary 2.2. Let $E$ be a normed space and let $F$ be a Banach space. Suppose $f: E \times E \rightarrow F$ is a mapping with $f(0, y)=0$ and there exist constants $\epsilon, \varepsilon, \theta$, $\vartheta \geq 0$ and $p \in[0,5)$ such that

$$
\begin{aligned}
\| f\left(a x_{1}+b x_{2}, y\right)+f\left(a x_{1}-b x_{2}, y\right)-2 a^{2} f\left(x_{1}, y\right) & \\
-2 b^{2} f\left(x_{2}, y\right) \| \leq & \epsilon+\theta\left(\left\|x_{1}\right\|^{p}+\left\|x_{2}\right\|^{p}+\|y\|^{p}\right), \\
\| f\left(x, a y_{1}+b y_{2}\right)+f\left(x, a y_{1}-b y_{2}\right)-a b^{2} f\left(x, y_{1}+y_{2}\right) & \\
-a b^{2} f\left(x, y_{1}-y_{2}\right)-2 a\left(a^{2}-b^{2}\right) f\left(x, y_{1}\right) \| \leq & \leq+\vartheta\left(\|x\|^{p}+\left\|y_{1}\right\|^{p}+\left\|y_{2}\right\|^{p}\right),
\end{aligned}
$$


for all $x, x_{1}, x_{2}, y, y_{1}, y_{2} \in E$. Then there exists a unique quintic mapping $j: E \rightarrow F$ such that

$$
\|f(x, y)-j(x, y)\| \leq \frac{\epsilon+\theta\left(\|x\|^{p}+\|y\|^{p}\right)}{a^{2}-a^{p-3}}+\frac{\varepsilon+\vartheta\left(\|a x\|^{p}+\|y\|^{p}\right)}{a^{5}-a^{p}},
$$

for all $x, y \in E$ and $a \in \mathbb{Z}_{+} \backslash\{1\}$.

Proof. It is known that every normed space is modular space with the modular $\rho(x)=\|x\|$ and $\kappa=2$. Define

$\phi\left(x_{1}, x_{2}, y\right)=\epsilon+\theta\left(\left\|x_{1}\right\|^{p}+\left\|x_{2}\right\|^{p}+\|y\|^{p}\right), \quad \psi\left(x, y_{1}, y_{2}\right)=\varepsilon+\vartheta\left(\|x\|^{p}+\left\|y_{1}\right\|^{p}+\left\|y_{2}\right\|^{p}\right)$,

and apply Theorem 2.1 .

\section{References}

[1] J. Aczel and J. Dhombres, Functional Equations in Several Variables, Cambridge University Press, Cambridge, 1989.

[2] T. Aoki, On the stability of the linear transformation in Banach spaces, J. Math. Soc. Japan., 2(1950), 64-66.

[3] P. W. Cholewa, Remarks on the stability of functional equations, Aequat. Math., 27(1984), 76-86.

[4] J. K. Chung and P. K. Sahoo, On the general solution of a quartic functional equation, Bull. Korean Math. Soc., 40(2003), 565-576.

[5] S. Czerwik, On the stability of the quadratic mapping in normed spaces, Abh. Math. Sem. Univ. Hamburg., 62(1992), 59-64.

[6] S. Czerwik, Functional Equations and Inequalities in Several Variables, World Scientific, London, 2002.

[7] A. Ebadian, A. Najati and M. E. Gordji, On approximate additive-quartic and quadratic-cubic functional equations in two variables on abelian groups, Results. Math., DOI 10.1007/s00025-010-0018-4 (2010)

[8] A. Ebadian, N. Ghobadipour and M. E. Gordji, A fixed point method for perturbation of bimultipliers and Jordan bimultipliers in $C^{*}$-ternary algebras, J. Math. Phys., 51 (2010), 10 pages, doi:10.1063/1.3496391.

[9] Z. Gajda, On stability of additive mappings, Internat. J. Math. Math. Sci., 14(1991), 431-434.

[10] P. Găvruta, A generalization of the Hyers-Ulam-Rassias stability of approximately additive mappings, J. Math. Anal. Appl., 184(1994), 431-436.

[11] P. Găvruta and L. Găvruta, A new method for the generalized Hyers-Ulam-Rassias stability, Int. J. Nonlinear Anal. Appl., 1(2010), 11-18.

[12] M. B. Ghaemi, M. E. Gordji and H. Majani, Approximately quintic and sextic mappings on the probabilistic normed spaces, Bull. Korean Math. Soc., 47(2)(2012), 339352 . 
[13] M. E. Gordji, Stability of a functional equation deriving from quartic and additive functions, Bull. Korean Math. Soc., 47(2010), 491-502.

[14] M. Eshaghi Gordji, Y. J. Cho, M. B. Ghaemi and H. Majani, Approximately quintic and sextic mappings from r-divisible groups into Serstnev probabilistic Banach spaces: fixed point method, Discrete Dynamics in Nature and Society, 2011(2011), Article ID 572062, 16 pages.

[15] M. E. Gordji and M. B. Savadkouhi, Stability of a mixed type cubic-quartic functional equation in non-Archimedean spaces, Appl. Math. Lett., 23(2010), 1198-1202.

[16] M. E. Gordji, M. B. Ghaemi, S. K. Gharetapeh, S. Shams and A. Ebadian, On the stability of $J^{*}$-derivations, J. Geom. Phys., 60(2010), 454-459.

[17] M. E. Gordji, S. K. Gharetapeh, C. Park and S. Zolfaghri, Stability of an additivecubic-quartic functional equation, Advances in Differ. Equat., Vol. 2009, Article ID 395693, 20. pages.

[18] M. E. Gordji, S. K. Gharetapeh, J. M. Rassias and S. Zolfaghari, Solution and stability of a mixed type additive, quadratic and cubic functional equation, Advances in differ. equat., Vol. 2009, Article ID 826130, 17 pages, doi:10.1155/2009/826130.

[19] M. E. Gordji and H. Khodaei, Solution and stability of generalized mixed type cubic, quadratic and additive functional equation in quasi-Banach spaces, Nonlinear Anal., 71(2009), 5629-5643.

[20] M. E. Gordji and H. Khodaei, On the generalized Hyers-Ulam-Rassias stability of quadratic functional equations, Abstr. Appl. Anal., Vol. 2009, Article ID 923476, 11 pages.

[21] M. E. Gordji and H. Khodaei, The fixed point method for fuzzy approximation of a functional equation associated with inner product spaces, Discr. Dynam. in Nature and Soc., Vol. 2010, Article ID 140767, 15 pages, doi:10.1155/2010/140767.

[22] M. E. Gordji, H. Khodaei and R. Khodabakhsh, General quartic-cubic-quadratic functional equation in non-Archimedean normed spaces, U. P. B. Sci. Bull., Series A, 72(2010), 69-84.

[23] M. E. Gordji and A. Najati, Approximately $J^{*}$-homomorphisms: A fixed point approach, J. Geom. Phys., 60(2010), 809-814.

[24] D. H. Hyers, On the stability of the linear functional equation, Proc. Natl. Acad. Sci. USA, 27(1941), 222-224.

[25] D. H. Hyers and Th. M. Rassias, Approximate homomorphisms, Aeq. Math., 44(1992), 125-153.

[26] D. H. Hyers, G. Isac and Th. M. Rassias, Stability of Functional Equations in Several Variables, Birkhäuser, Basel, 1998.

[27] G. Isac and Th. M. Rassias, On the Hyers-Ulam stability of $\psi$-additive mappings, J. Approx. Theory, 72(1993), 131-137.

[28] G. Isac and Th. M. Rassias, Stability of $\psi$-additive mappings : Applications to non linear analysis, Internat. J. Math. and Math. Sci., 19(2)(1996), 219-228.

[29] K. W. Jun and H. M. Kim, The generalized Hyers-Ulam-Rassias stability of a cubic functional equation, J. Math. Anal. Appl., 274(2002), 867-878. 
[30] K. W. Jun, H. M. Kim and I. S. Chang, On the Hyers-Ulam stability of an EulerLagrange type cubic functional equation, J. Comput. Anal. Appl., 7(2005), 21-33.

[31] S. M. Jung, Hyers-Ulam-Rassias Stability of Functional Equations in Mathematical Analysis, Hadronic Press Inc., Palm Harbor, Florida, 2001.

[32] S. M. Jung, Hyers-Ulam-Rassias stability of Jensen's equation and its application, Proc. Amer. Math. Soc., 126(1998), 3137-3143.

[33] S. M. Jung, Stability of the quadratic equation of Pexider type, Abh. Math. Sem. Univ. Hamburg, 70(2000), 175-190.

[34] P. Kannappan, Quadratic functional equation and inner product spaces, Results Math., 27(1995), 368-372.

[35] M. A. Khamsi, Quasicontraction Mapping in modular spaces without $\Delta_{2}$-condition, Fixed Point Theory and Applications Volume (2008), Artical ID 916187, 6 pages.

[36] H. Khodaei and Th. M. Rassias, Approximately generalized additive functions in several variables, Int. J. Nonlinear Anal. Appl., 1(2010), 22-41.

[37] S. Koshi, T. Shimogaki, On F-norms of quasi-modular spaces, J. Fac. Sci. Hokkaido Univ. Ser. I, 15(3)(1961), 202-218.

[38] M. Krbec, Modular interpolation spaces, Z. Anal. Anwendungen, 1(1982), 25-40.

[39] S. H. Lee, S. M. Im and I. S. Hawng, Quartic functional equation, J. Math. Anal. Appl., 307(2005), 387-394.

[40] W. A. Luxemburg, Banach function spaces, Ph. D. thesis, Delft Univrsity of technology, Delft, The Netherlands, 1959.

[41] L. Maligranda, Orlicz Spaces and Interpolation, in: Seminars in Math., Vol. 5, Univ. of Campinas, Brazil, 1989.

[42] J. Musielak, Orlicz Spaces and Modular Spaces, in: Lecture Notes in Math. Vol. 1034, Springer-verlag, Berlin, 1983.

[43] A. Najati, Hyers-Ulam-Rassias stability of a cubic functional equation, Bull. Korean Math. Soc., 44(2007), 825-840.

[44] H. Nakano, Modulared Semi-Ordered Linear Spaces, in: Tokyo Math. Book Ser., Vol. 1, Maruzen Co., Tokyo, 1950.

[45] W. Orlicz, Collected Papers, Vols. I, II, PWN, Warszawa, 1988.

[46] C. Park, On an approximate automorphism on a $C^{*}$-algebra, Proc. Amer. Math. Soc., 132(2004), 1739-1745.

[47] C. Park and M. E. Gordji, Comment on Approximate ternary Jordan derivations on Banach ternary algebras [Bavand Savadkouhi et al. J. Math. Phys. 50, 042303 (2009)], J. Math. Phys. 51, 044102 (2010), 7 pages.

[48] C. Park and A. Najati, Generalized additive functional inequalities in Banach algebras, Int. J. Nonlinear Anal. Appl., 1(2010), 54-62.

[49] C. Park and Th. M. Rassias, Isomorphisms in unital $C^{*}$-algebras, Int. J. Nonlinear Anal. Appl., 1(2010), 1-10.

[50] C. Park and J. M. Rassias, Stability of the Jensen-type functional equation in $C^{*}$ algebras: a fixed point approach, Abstr. Appl. Anal. Vol. 2009, Article ID 360432, 17 pages. 
[51] Th. M. Rassias, On the stability of the linear mapping in Banach spaces, Proc. Amer. Math. Soc., 72(1978), 297-300.

[52] Th. M. Rassias, On the stability of functional equations in Banach spaces, J. Math. Anal. Appl., 251(1)(2000), 264-284.

[53] Th. M. Rassias and P. Šemrl, On the behaviour of mappings which do not satisfy Hyers-Ulam stability, Proc. Amer. Math. Soc., 114(1992), 989-993.

[54] Gh. Sadeghi, A fixed point approach to stability of functional equations in modular spaces, Bull. Malays. Math. Sci. Soc., to appear.

[55] Gh. Sadeghi, On the orthogonal stability of the pexiderized quadratic equations in modular spaces, preprint.

[56] F. Skof, Propriet locali e approssimazione di operatori, Rend. Sem. Mat. Fis. Milano., 53(1983), 113-129.

[57] Ph. Turpin, Fubini inequalities and bounded multiplier property in generalized modular spaces, Comment. Math., Tomus specialis in honorem Ladislai Orlicz I (1978), 331353.

[58] S. M. Ulam, Problems in Modern Mathematics, Chapter VI, Sci. Ed., Wiley, New York, 1964.

[59] S. Yamamuro, On conjugate spaces of Nakano spaces, Trans. Amer. Math. Soc., 90(1959), 291-311. 